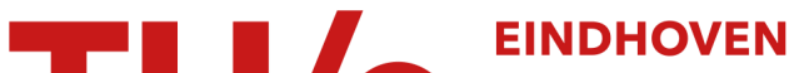 UNIVERSITY OF TECHNOLOGY
}

\section{Tracking control for networked control systems}

\section{Citation for published version (APA):}

Wouw, van de, N., Naghshtabrizi, P., Posthumus - Cloosterman, M. B. G., \& Hespanha, J. P. (2007). Tracking control for networked control systems. In Proceedings of the 46th IEEE Conference on Decision and Control (CDC 46), 12-14 December 2007, New Orleans, USA (pp. 4441-4446). Institute of Electrical and Electronics Engineers. https://doi.org/10.1109/CDC.2007.4434905

DOI:

10.1109/CDC.2007.4434905

Document status and date:

Published: 01/01/2007

\section{Document Version:}

Publisher's PDF, also known as Version of Record (includes final page, issue and volume numbers)

\section{Please check the document version of this publication:}

- A submitted manuscript is the version of the article upon submission and before peer-review. There can be important differences between the submitted version and the official published version of record. People interested in the research are advised to contact the author for the final version of the publication, or visit the $\mathrm{DOI}$ to the publisher's website.

- The final author version and the galley proof are versions of the publication after peer review.

- The final published version features the final layout of the paper including the volume, issue and page numbers.

Link to publication

\section{General rights}

Copyright and moral rights for the publications made accessible in the public portal are retained by the authors and/or other copyright owners and it is a condition of accessing publications that users recognise and abide by the legal requirements associated with these rights.

- Users may download and print one copy of any publication from the public portal for the purpose of private study or research.

- You may not further distribute the material or use it for any profit-making activity or commercial gain

- You may freely distribute the URL identifying the publication in the public portal.

If the publication is distributed under the terms of Article 25fa of the Dutch Copyright Act, indicated by the "Taverne" license above, please follow below link for the End User Agreement:

www.tue.nl/taverne

Take down policy

If you believe that this document breaches copyright please contact us at:

openaccess@tue.nl

providing details and we will investigate your claim. 


\title{
Tracking Control for Networked Control Systems
}

\author{
Nathan van de Wouw, Payam Naghshtabrizi, Marieke Cloosterman, João Pedro Hespanha
}

\begin{abstract}
A solution to the approximate tracking problem for Networked Control Systems (NCSs) with uncertain, timevarying sampling intervals and network delays is presented. The uncertain, time-varying sampling and delays cause inexact feedforward, which induces a perturbation on the tracking error dynamics. Two alternative modeling approaches are used: a discrete-time model and a model in terms of delay impulsive differential equations. Sufficient conditions for the input-tostate stability (ISS) of the tracking error dynamics with respect to this perturbation are given. These ISS results provide bounds on the steady-state tracking error as a function of the plant properties, the controller parameters, and the network properties. The results are illustrated on a mechanical motion control example.
\end{abstract}

\section{INTRODUCTION}

In this paper, we study the tracking control problem for NCSs with uncertain, time-varying sampling intervals and network delays. NCSs are control systems in which the communication between the actuators, sensors and the controllers takes place over a communication network. The benefits of using such a communication network, as opposed to dedicated point-to-point wiring, is increased architectural flexibility, decreased maintenance costs and system wiring [1], [2]. The presence of the communication network; however, induces non-ideal behavior in the form of uncertain, and time-varying sampling intervals, network delays, and packet loss [1].

Different models for NCSs with uncertain, and timevarying sampling intervals and network delays have been proposed in literature. In [3], [4], [5], [6], a discrete-time modeling approach is employed in the face of network delays. In [7], NCSs, with uncertain sampling intervals and network delays, are modeled in terms of impulsive delay differential equations. For both model-types, stability criteria have been proposed; see [5], [6] for stability conditions for discrete-time models and to [7] for stability results for the impulsive delay models.

To this date, the work on NCSs has largely focused on modeling, stability, and stabilization problems. Tracking control, however, poses additional challenges, some of which are specifically aggravated by the network. In tracking control, typical high-performance designs include

Nathan van de Wouw and Marieke Cloosterman are with the Department of Mechanical Engineering, Eindhoven University of Technology, POBox 513, 5600 MB Eindhoven, The Netherlands n.v.d.wouw@tue.nl,M.B.G.Cloostermandtue.nl

Cloosterman was supported by the Boderc project under the responsibility of the Embedded Systems Institute. This project is partially supported by the Dutch Ministry of Economic Affairs under the Senter TS program.

Payam Naghshtabrizi and João Hespanha are with the Department of Electrical \& Computer Engineering, University of California, Santa Barbara, CA 93106-9560, U.S.A., payam@ece. ucsb. edu, hespanhadece.ucsb.edu

Naghshtabrizi and Hespanha were supported by the Institute for Collaborative Biotechnologies through grant DAAD19-03-D-0004 from the U.S. Army Research Office and by the National Science Foundation under Grant No. CCR-0311084. feedforward thereby inducing the desired trajectory in the controlled system, whereas feedback assures convergence to the desired solution and favorable robustness and disturbance attenuation properties. Due to the delays and variation in sampling intervals, the feedforward signal generally arrives at the actuator later than intended, leading to a networkinduced feedforward error and reduced tracking performance. Consequently, only approximate tracking can be achieved. In the NCS literature, the tracking problem has received little attention. Recent works related to the tracking control of NCSs are [8], [9]. In [8], an $H_{\infty}$-approach towards the tracking control problem of NCSs with network delays (and constant sampling intervals) is presented; however, the fact that the feedforward generally experiences delays is not taken into account. In [9], the optimal tracking control problem is studied with a focus on the effects of quantization of the feedforward.

Here, we analyze the ISS property of NCSs with respect to the feedforward error. Based on the ISS property we provide an asymptotic upper bound for the tracking error that depends on the properties of the plant, the controller and the network. ISS properties of (nonlinear) NCSs have been studied in [10]. Herein, the role of the network protocol in guaranteeing such stability properties is studied and NCSs with time-varying sampling intervals and multiple-packet communication are considered; however, no network delays are taken into account.

The outline of the paper is as follows. In Section II, an NCS model for tracking is proposed and the approximate tracking problem is formulated. In Section III, two approaches for analyzing the ISS properties of this NCS model are addressed. Moreover, ultimate bounds for the tracking error are provided in Section IV. In Section V, an example is presented illustrating the benefit of the ISS results. Finally, conclusions are given in Section VI. Most proofs will be omitted due to lack of space and can be found in an extended version of this paper [11].

Notation: A function $\gamma:[0, \infty) \rightarrow[0, \infty)$ is said to be of class $-\mathcal{G}$ if it is continuous, zero at zero and non-decreasing. It is of class- $\mathcal{K}$ if it is of class- $\mathcal{G}$ and strictly increasing. It is of class- $\mathcal{K}_{\infty}$ if it is of class- $\mathcal{K}$ and unbounded. A continuous function $\beta:[0, \infty) \times[0, \infty) \rightarrow[0, \infty)$ is said to be of class$\mathcal{K} \mathcal{L}$ if $\beta(., t)$ is of class- $\mathcal{K}$ for each $t \geq 0$ and $\beta(s,$.$) is$ monotonically decreasing to zero for each $s>0$. We denote the transpose of a matrix $\boldsymbol{A}$ by $\boldsymbol{A}^{T}$ and we write $\boldsymbol{P}>0$ (or $\boldsymbol{P}<0$ ) when $P$ is a symmetric positive (or negative) definite matrix. We write a symmetric matrix $\left[\begin{array}{cc}\boldsymbol{A} & \boldsymbol{B} \\ \boldsymbol{B}^{T} & \boldsymbol{C}\end{array}\right]$ as $\left[\begin{array}{cc}\boldsymbol{A} & \boldsymbol{B} \\ * & \boldsymbol{C}\end{array}\right]$. When there is no confusion we write $\boldsymbol{x}(t)$ as $\boldsymbol{x}$.

\section{AN NCS MODEL FOR TRACKING CONTROL}

A two-channel NCS is schematically depicted in Figure 1. It consists of a continuous-time plant and a discrete-time 


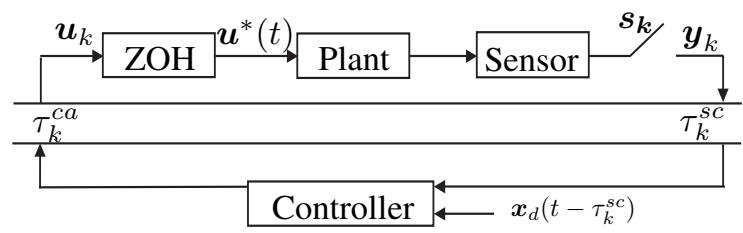

Figure 1: Schematic overview of the two-channel NCS with variable sampling and network delays.

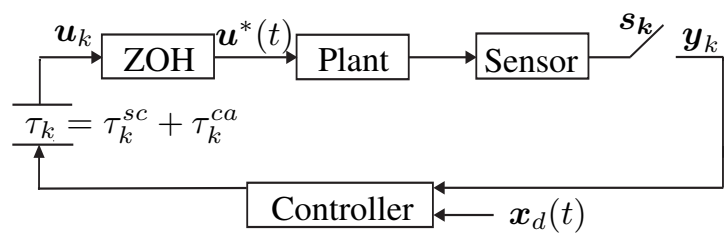

Figure 2: Schematic overview of the one-channel NCS with variable sampling and network delays.

controller, which receives information from the plant only at the sampling instants $s_{k}$. Due to the fact that we allow for a variable sampling interval $h_{k}$, the samplings instants $s_{k}=\sum_{i=1}^{k} h_{i}, \quad \forall k \geq 1, s_{0}=0$, are non-equidistantly spaced. Moreover, the computation times and the network delays result in a sensor-to-controller delay $\tau_{k}^{s c}$ and in a controller-to-actuator delay $\tau_{k}^{c a}$, which have to be taken into account. Similar to [1], the sensor acts in a time-driven (though variable) fashion and the controller and actuator (including the zero-order-hold ( $\mathrm{ZOH})$ in Figure 1) act in an event-driven fashion. Hereto, we assume that all sensors are sampled synchronously and there is a single sensor-sending source.

The two-channel NCS in Figure 1 is equivalent to the one-channel NCS in Figure 2 with $\tau_{k}:=\tau_{k}^{s c}+\tau_{k}^{c a}$ given that the controller is static and time-invariant [4], [1]. We call $\tau_{k}$ the ( $k^{t h}$ total) loop delay and $t_{k}:=s_{k}+\tau_{k}$ the $\left(k^{t h}\right)$ input update time. Out of order packets will be dropped and consequently the sampling times $\left\{s_{1}, s_{2}, s_{3}, \cdots\right\}$ and the input update times $\left\{t_{1}, t_{2}, t_{3}, \cdots\right\}$ form strictly increasing sequences in $\left[s_{0}, \infty\right)$ for some initial time $s_{0}$.

The continuous-time model of the plant is given by:

$$
\begin{aligned}
& \dot{\boldsymbol{x}}(t)=\boldsymbol{A x}(t)+\boldsymbol{B} \boldsymbol{u}^{*}(t), \quad \boldsymbol{x}(0)=\boldsymbol{x}_{0}, \\
& \boldsymbol{u}^{*}(t)=\boldsymbol{u}_{k}, \quad \text { for } t \in\left[t_{k}, t_{k+1}\right), \quad t_{1}=\tau_{1},
\end{aligned}
$$

with $\boldsymbol{A}$ and $\boldsymbol{B}$ the system and input matrices, $t \in \mathbb{R}$ the time variable, $\boldsymbol{u}_{k}=\boldsymbol{u}\left(s_{k}\right) \in \mathbb{R}^{m}$ the sampled input, and $\boldsymbol{x}_{k}=\boldsymbol{x}\left(s_{k}\right) \in \mathbb{R}^{n}$ the state at sampling times.

In what follows we introduce the tracking problem, control signal construction, the tracking error dynamics, and we argue that the ISS property of the error dynamics is the relevant notion to study the effect of the network on the tracking problem.

Control signal construction: We desire the system to asymptotically track a desired trajectory $\boldsymbol{x}_{d}(t)$. The proposed control law consists of a feedforward part and a feedback part. The exact feedforward $\boldsymbol{u}_{e}^{f f}(t)$ should be selected such that the desired state trajectory $\boldsymbol{x}_{d}(t)$ is a solution to the continuous-time system

$$
\dot{\boldsymbol{x}}_{d}(t)=\boldsymbol{A} \boldsymbol{x}_{d}(t)+\boldsymbol{B} \boldsymbol{u}_{e}^{f f}(t) .
$$

Here, we assume that $\boldsymbol{x}_{d}(t)$ is at least $\mathcal{C}^{2}$, guaranteeing that $\boldsymbol{u}_{e}^{f f}(t)$ is at least $\mathcal{C}^{1}$. We propose the following tracking control law for (1):

$$
\boldsymbol{u}_{k}:=\boldsymbol{u}_{e}^{f f}\left(s_{k}\right)-\boldsymbol{K}\left(\boldsymbol{x}_{k}-\boldsymbol{x}^{d}\left(s_{k}\right)\right)
$$

that consists of the superposition of a sampled feedforward component $\boldsymbol{u}_{e}^{f f}\left(s_{k}\right)$ with a linear tracking-error feedback component with feedback gain matrix $\boldsymbol{K} \in \mathbb{R}^{m \times n}$. We employ time-stamping on the measurements; so, the sampling time $s_{k}$ is known, which enables the computation of the control command (3) at time $t_{k}:=s_{k}+\tau_{k}$.

The implemented continuous-time feedforward $\boldsymbol{u}^{f f}(t)$ in (1), (3) is piecewise constant, given by

$$
\boldsymbol{u}^{f f}(t)=\boldsymbol{u}_{e}^{f f}\left(s_{k}\right) \text { for } t \in\left[t_{k}, t_{k+1}\right),
$$

and differs from the exact feedforward $\boldsymbol{u}_{e}^{f f}(t)$ due to the zero-order hold and the network delays. Therefore, we will decompose the implemented feedforward as the sum of the exact feedforward part $\boldsymbol{u}_{e}^{f f}(t)$ and a feedforward error: $\boldsymbol{u}^{f f}(t)=\boldsymbol{u}_{e}^{f f}(t)+\boldsymbol{\Delta} \boldsymbol{u}^{f f}(t)$ for $t \in\left[t_{k}, t_{k+1}\right)$, with the feedforward error simply defined by $\boldsymbol{\Delta} \boldsymbol{u}^{f f}(t)=\boldsymbol{u}_{e}^{f f}\left(s_{k}\right)$ $\boldsymbol{u}_{e}^{f f}(t)$ for $t \in\left[t_{k}, t_{k+1}\right)$.

Closed-loop system: Applying the control law (3) to system (1) yields the following closed-loop NCS dynamics: $\dot{\boldsymbol{x}}(t)=\boldsymbol{A} \boldsymbol{x}(t)+\boldsymbol{B}_{1}\left(\boldsymbol{x}_{k}-\boldsymbol{x}^{d}\left(s_{k}\right)\right)+\boldsymbol{B}_{2}\left(\boldsymbol{u}_{e}^{f f}(t)+\boldsymbol{\Delta} \boldsymbol{u}^{f f}(t)\right)$,

for $t \in\left[t_{k}, t_{k+1}\right)$ and with $\boldsymbol{B}_{1}:=-\boldsymbol{B} \boldsymbol{K}$ and $\boldsymbol{B}_{2}:=\boldsymbol{B}$. The initial condition $\overline{\boldsymbol{x}}(0):=\left[\begin{array}{ll}\boldsymbol{x}^{T}(0) & \boldsymbol{x}^{T}\left(s_{0}\right)\end{array}\right]^{T}$ for this system consists of both the initial state at time $s_{1}=0$, i.e., $\boldsymbol{x}(0)=\boldsymbol{x}_{0}$, and the hold state $\boldsymbol{x}\left(s_{0}\right)$ at time $s_{0}<0$. So, the network delays cause the initial state to involve a past state.

Tracking error dynamics: We define the tracking error $\boldsymbol{e}$ by $\boldsymbol{e}=\boldsymbol{x}-\boldsymbol{x}^{d}$. By combining (5) and (2) we can formulate the continuous-time tracking error dynamics as follows:

$$
\dot{\boldsymbol{e}}(t)=\boldsymbol{A} \boldsymbol{e}(t)+\boldsymbol{B}_{1} \boldsymbol{e}\left(s_{k}\right)+\boldsymbol{B}_{2} \boldsymbol{\Delta} \boldsymbol{u}^{f f}(t),
$$

for $t \in\left[t_{k}, t_{k+1}\right)$, and with $\overline{\boldsymbol{e}}(0):=\left[\begin{array}{ll}\boldsymbol{e}^{T}(0) & \boldsymbol{e}^{T}\left(s_{0}\right)\end{array}\right]^{T}$.

We consider the approximate tracking problem. Herein, we aim to ensure asymptotic ultimate boundedness of the tracking error, i.e. $\boldsymbol{e}(t)=\boldsymbol{x}(t)-\boldsymbol{x}^{d}(t) \leq \epsilon$ for $t \rightarrow \infty$ for some small $\epsilon>0$. Some tracking error is to be expected in the NCS setting, as the implemented feedforward signal $\boldsymbol{u}^{f f}(t)$ in (4) will never equal the exact feedforward $\boldsymbol{u}_{e}^{f f}(t)$. The reasons for non-exact feedforward are, firstly, the fact that the control signal (and therefore also the feedforward signal) will be passed through a zero-order hold and, secondly, the fact that the network delays (in particular the controllerto-actuator delay $\tau_{k}^{c a}$ ) in general cause the feedforward to be implemented too late. In the next section, we will propose sufficient conditions for the input-to-state stability (ISS) of the continuous-time tracking error dynamics (6) with respect to the input $\boldsymbol{\Delta} \boldsymbol{u}^{f f}(t)$. An ISS property of the tracking error dynamics guarantees that the controller solves an approximate tracking problem.

\section{INPUT-TO-STATE STABILITY OF NCSS WITH TIME-VARYING DELAYS AND SAMPLE TIMES}

In this section, we propose sufficient conditions for the input-to-state stability of the continuous-time tracking error dynamics (6) with respect to the input $\boldsymbol{\Delta} \boldsymbol{u}^{f f}(t)$. In the 
subsequent subsections, we propose two approaches towards proving such ISS properties: in the first approach, the dynamics are largely analyzed in a discrete-time setting, whereas in the second approach the dynamics are analyzed using delay impulsive differential equations. We will see later that, depending on the problem, either approach can be favorable over the other when considering the stability bounds and the ISS gains provided.

We say that the system (6) is uniformly ISS over a given class $\mathcal{S}$ of admissible sequences of sampling times and delays $\left\{s_{k}, \tau_{k}\right\}$ if there exist a $\mathcal{K} \mathcal{L}$-function $\beta(r, s)$ and a $\mathcal{K}$-function $\gamma(r)$ such that, for any initial condition $\overline{\boldsymbol{e}}(0)$ and any bounded input $\boldsymbol{\Delta} \boldsymbol{u}^{f f}(t)$, the solution to (6) satisfies

$$
|\boldsymbol{e}(t)| \leq \beta(|\overline{\boldsymbol{e}}(0)|, t)+\gamma\left(\sup _{0 \leq s \leq t}\left|\boldsymbol{\Delta} \boldsymbol{u}^{f f}(s)\right|\right),
$$

with functions $\beta$ and $\gamma$ that are independent of the choice of the particular sequence $\left\{s_{k}, \tau_{k}\right\}$. We would like to have the ISS property for any sequence of delays such that $\tau_{\min } \leq \tau_{k} \leq \tau_{\max }, \forall k \in \mathbb{N}$ and any sequence of sampling times such that $h_{\min } \leq s_{k+1}-s_{k} \leq h_{\max }$ for given $h_{\min }, h_{\max }, \tau_{\min }, \tau_{\max }$ where $0 \leq h_{\min } \leq h_{\max }$ and $0 \leq \tau_{\min } \leq \tau_{\max }$. Consequently, the class of admissible sequences is characterized by

$$
\begin{array}{r}
\mathcal{S}:=\left\{\left\{s_{k}, \tau_{k}\right\}: h_{\min } \leq s_{k+1}-s_{k} \leq h_{\max },\right. \\
\left.\tau_{\min } \leq \tau_{k} \leq \tau_{\max }\right\} .
\end{array}
$$

\section{A. Discrete-time Approach}

Under the assumption that $\tau_{k}<h_{k}, \forall k$, the discretization of (6) at the sampling instants $s_{k}$ results in the following discrete-time system, which forms the basis of our analysis:

$$
\begin{aligned}
\boldsymbol{e}_{k+1}= & e^{\boldsymbol{A} h_{k}} \boldsymbol{e}_{k}+\int_{0}^{h_{k}-\tau_{k}} e^{\boldsymbol{A} s} d s \boldsymbol{B}_{1} \boldsymbol{e}_{k} \\
& +\int_{h_{k}-\tau_{k}}^{h_{k}} e^{\boldsymbol{A} s} d s \boldsymbol{B}_{1} \boldsymbol{e}_{k-1}+\overline{\boldsymbol{\Delta}}_{k}^{f f},
\end{aligned}
$$

where $\overline{\boldsymbol{\Delta}}_{k}^{f f}:=\int_{0}^{h_{k}} e^{\boldsymbol{A}_{s}} \boldsymbol{B}_{2} \boldsymbol{\Delta} \boldsymbol{u}^{f f}\left(h_{k}+s_{k}-s\right) d s$. Since $\tau_{k}<h_{k}, \forall k$, we can define an extended state for the system (9) by $\boldsymbol{\xi}_{k}:=\left(\begin{array}{ll}\boldsymbol{e}_{k}^{T} & \boldsymbol{e}_{k-1}^{T}\end{array}\right)^{T}$ and we obtain the following discrete-time state-space model:

$$
\boldsymbol{\xi}_{k+1}=\tilde{\boldsymbol{A}} \boldsymbol{\xi}_{k}+\tilde{\boldsymbol{B}} \overline{\boldsymbol{\Delta u}}_{k}^{f f}
$$

with $\boldsymbol{\xi}_{k} \in \mathbb{R}^{2 n}$,

$$
\tilde{\boldsymbol{A}}=\left[\begin{array}{cc}
e^{\boldsymbol{A} h_{k}}+\int_{0}^{h_{k}-\tau_{k}} e^{\boldsymbol{A} s} d s \boldsymbol{B}_{1} & \int_{h_{k}-\tau_{k}}^{h_{k}} e^{\boldsymbol{A} s} d s \boldsymbol{B}_{1} \\
\boldsymbol{I} & \mathbf{0}
\end{array}\right]
$$

and $\tilde{\boldsymbol{B}}^{T}=\left[\begin{array}{ll}\boldsymbol{I} & \mathbf{0}\end{array}\right]$, where $\tilde{\boldsymbol{A}}=\tilde{\boldsymbol{A}}\left(h_{k}, \tau_{k}\right)$. Moreover, $\boldsymbol{e}_{k}=$ $\boldsymbol{C}_{z} \boldsymbol{\xi}_{k}$, with $\boldsymbol{C}_{z}=\left[\begin{array}{ll}\boldsymbol{I} & \mathbf{0}\end{array}\right]$. Note that the matrix $\tilde{\boldsymbol{A}}$ depends on the $s_{k}$ and $\tau_{k}$, but this dependence was not made explicit to keep the formulas short.

Before we formulate conditions for the input-to-state stability of system (6), we recall results on the global asymptotic stability of the equilibrium point $\boldsymbol{\xi}=\mathbf{0}$ of the discretetime system (10) for the case that $\overline{\boldsymbol{\Delta u}}_{k}^{f f}=\mathbf{0}$ for all $k$ (i.e., the case of stabilization). When $\bar{\Delta}_{k}^{f f}=\mathbf{0}$, the system (10) can be viewed as a switching discrete-time system for which stability can be guaranteed using a common quadratic Lyapunov function approach. More specifically, stability is guaranteed if the following (infinite) set of matrix inequalities is feasible:

$$
\tilde{\boldsymbol{A}}^{T} \boldsymbol{P} \tilde{\boldsymbol{A}}-(1-\alpha) \boldsymbol{P}<0, \quad \boldsymbol{P}=\boldsymbol{P}^{T}>0
$$

$\forall\left\{s_{k}, \tau_{k}\right\} \in \mathcal{S}$ and for some $0<\alpha<1$. Based on these stability results, we will show (see Theorem 1) that the inputto-state stability of (6) is guaranteed if the following (infinite) set of matrix inequalities is feasible:

$$
\begin{gathered}
\boldsymbol{P}=\boldsymbol{P}^{T}>0 \\
{\left[\begin{array}{cc}
\tilde{\boldsymbol{A}}^{T} \boldsymbol{P} \tilde{\boldsymbol{A}}-(1-\alpha) \boldsymbol{P} & \tilde{\boldsymbol{A}}^{T} \boldsymbol{P} \tilde{\boldsymbol{B}} \\
* & \tilde{\boldsymbol{B}}^{T} \boldsymbol{P} \tilde{\boldsymbol{B}}-c_{4} \boldsymbol{I}
\end{array}\right]<0}
\end{gathered}
$$

$\forall\left\{s_{k}, \tau_{k}\right\} \in \mathcal{S}$, for some $0<\alpha<1$ and $c_{4}>0$. For the case of constant sampling intervals $h_{k}=h, \forall k$, sufficient conditions for the feasibility of (12) in terms of (finite sets) of LMIs are proposed in [5] based on a (real) Jordan form representation of the NCS applicable to both small and large delays. Based on the sufficiency of (13) for ISS, the necessary derivations of the finite set of LMIs are similar to those in [5]. For the sake of brevity, we will omit such technicalities here.

Let us now present the result on the input-to-state stability of the continuous-time tracking error dynamics (6).

\section{Theorem 1}

Consider the tracking error dynamics (6) with $\left\{s_{k}, \tau_{k}\right\} \in \mathcal{S}$ and $\tau_{k}<s_{k+1}-s_{k}, \forall k$. Suppose there exist a matrix $\boldsymbol{P}$ and scalars $0<\alpha<1$ and $c_{4}>0$ for which (13) is satisfied. Then, the system (6) is uniformly ISS over the class $\mathcal{S}$ with respect to the time-varying input $\boldsymbol{\Delta} \boldsymbol{u}^{f f}(t)$ and the inequality (7) is satisfied with

$$
\beta(r, t)=g_{1}(t) r, \quad \gamma(r)=g_{2} r,
$$

where

$$
\begin{aligned}
g_{1}(t) & = \begin{cases}g_{1,1}+\epsilon+\frac{\epsilon}{h_{1}} t, & t \in\left[0, s_{2}\right] \\
g_{1, k-1}+\frac{g_{1, k}-g_{1, k-1}}{h_{k}}\left(t-s_{k}\right), & k \geq 2, t \in\left[s_{k}, s_{k+1}\right],\end{cases} \\
g_{1, k} & =\left\|\boldsymbol{C}_{z} \boldsymbol{P}^{-\frac{1}{2}}\right\|\left(c_{1} \sqrt{\bar{\alpha}^{k} \lambda_{\max }(\boldsymbol{P})}+c_{2} \sqrt{\bar{\alpha}^{k-1} \lambda_{\max }(\boldsymbol{P})}\right), \\
g_{2} & =c_{3}\left(1+\left(c_{1}+c_{2}\right)\left\|\boldsymbol{C}_{z} \boldsymbol{P}^{-\frac{1}{2}}\right\| \sqrt{\frac{c_{4}}{\alpha}}\right)
\end{aligned}
$$

with $g_{1, k}$ defined for $k \geq 1, \epsilon>0, \bar{\alpha}:=1-\alpha$ and

$$
\begin{aligned}
c_{1} & =\max \left(\bar{c}_{1}, \hat{c}_{1}+\hat{c}_{2}\right), c_{2}=\max \left(\bar{c}_{2}, \tilde{c}_{2}\right), \\
c_{3} & =\left\|\boldsymbol{B}_{2}\right\| c\left(h_{\max }, h_{\max }\right),
\end{aligned}
$$

$$
\begin{aligned}
& \text { with } c\left(r_{1}, r_{2}\right):=\left\{\begin{array}{ll}
\frac{e^{\lambda_{\max } r_{1}}-1}{\lambda_{\max }} & \text { if } \lambda_{\max } \neq 0 \\
r_{2} & \text { if } \lambda_{\max }=0
\end{array}\right. \text { and } \\
& \bar{c}_{1}=\max \left(1, e^{\lambda_{\max } \tau_{\max }}\right), \\
& \bar{c}_{2}=\left\|\boldsymbol{B}_{1}\right\| c\left(\tau_{\max }, \tau_{\max }\right), \tilde{c}_{2}=\left\|\boldsymbol{B}_{1}\right\| c\left(h_{\max }, \tau_{\max }\right) \\
& \hat{c}_{2}=\left\|\boldsymbol{B}_{1}\right\| c\left(h_{\max }-\tau_{\min }, h_{\max }-\tau_{\min }\right), \\
& \hat{c}_{1}= \begin{cases}\max \left(e^{\lambda_{\max } h_{\max }}, e^{\lambda_{\max } \tau_{\min }}\right) & \text { if } \lambda_{\max } \neq 0 \\
1 & \text { if } \lambda_{\max }=0 .\end{cases}
\end{aligned}
$$

This result implies that the state $e$ of the NCS is globally uniformly ultimately bounded and the asymptotic bound is given by $\lim \sup _{t \rightarrow \infty}|\boldsymbol{e}(t)| \leq g_{2} \sup _{t \geq 0}\left|\boldsymbol{\Delta} \boldsymbol{u}^{f f}(t)\right|$ with $g_{2}$ 
as in (15). Note that all parameters in (15), (16) and (17) are known and depend on the system dynamics and the feedback gain matrix (matrices $\boldsymbol{A}, \boldsymbol{B}_{1}$ and $\boldsymbol{B}_{2}$ ), the network parameters (maximum and minimum sampling intervals $h_{\max }$ and $h_{\text {min }}$, respectively, and maximum and minimum delays $\tau_{\max }$ and $\tau_{\text {min }}$, respectively) and the parameters $\alpha, c_{4}$ and matrix $\boldsymbol{P}$ satisfying (13).

An asymptotic bound for the tracking error $e$ at the sampling instants is given by

$$
\begin{aligned}
\limsup _{k \rightarrow \infty}\left|\boldsymbol{e}_{k}\right| & \leq c_{3}\left\|\boldsymbol{C}_{z} \boldsymbol{P}^{-\frac{1}{2}}\right\| \sqrt{\frac{c_{4}}{\alpha}} \sup _{t \geq 0}\left|\boldsymbol{\Delta} \boldsymbol{u}^{f f}(t)\right| \\
& =: \bar{g}_{2} \sup _{t \geq 0}\left|\boldsymbol{\Delta} \boldsymbol{u}^{f f}(t)\right| .
\end{aligned}
$$

This bound can in many practical cases (e.g., for sufficiently small sampling intervals) be sufficient and it is typically much less conservative since $\bar{g}_{2}<g_{2}$. The difference between $g_{2}$ and $\bar{g}_{2}$ originates from the need to upperbound the inter-sample behavior of $e$, thereby introducing additional conservatism.

\section{B. Delay Impulsive Approach}

In the previous section, we established sufficient conditions for the ISS property of the system (6) by adopting the discrete-time NCS modeling approach. As used in this paper, this approach required the delays to be smaller than the sampling interval, i.e., $\tau_{k} \leq h_{k}, \forall k$. Without this assumption, i.e., for large delays, the discrete-time approach yields increasingly complex models [12], [5]. We now model the system (6) as a delay impulsive system, which avoids an increase in model complexity when dealing with large delays.

Impulsive dynamical systems exhibit continuous evolutions described by ordinary differential equations and instantaneous state jumps or impulses. We refer to impulsive dynamical systems with delay in the jump equation as delay impulsive systems. First we consider a more general system of the form

$$
\begin{array}{ll}
\dot{\boldsymbol{x}}(t)=\boldsymbol{f}_{k}(\boldsymbol{x}(t), t, \boldsymbol{w}(t)), & t \in\left[t_{k}, t_{k+1}\right), \\
\boldsymbol{x}\left(t_{k+1}\right)=\boldsymbol{g}_{k}\left(\boldsymbol{x}\left(s_{k+1}^{-}\right), t_{k+1}^{-}\right), & k \in \mathbb{N},
\end{array}
$$

where $\boldsymbol{f}_{k}, \boldsymbol{g}_{k}$ are locally Lipschitz functions such that $\boldsymbol{f}_{k}(\mathbf{0}, t, \mathbf{0})=\mathbf{0}, \boldsymbol{g}_{k}(\mathbf{0}, t)=\mathbf{0}, \forall t \in \mathbb{R}_{\geq 0}$. For system (19), we assess the ISS property over the set $\mathcal{S}$ of impulse-delay sequences defined in (8) using the tools developed for delay differential equations in [13]. Given a Lyapunov-like function

$$
V: \mathbb{R}^{n} \times\left[0, \rho_{\max }\right] \times\left[-h_{\max }-\tau_{\max }, \infty\right) \rightarrow[0, \infty),
$$

we use the shorthand notation $V(t):=V(\boldsymbol{x}(t), \rho(t), t)$, where $\rho(t):=t-t_{k}, t \in\left[t_{k}, t_{k+1}\right)$ characterizes the time between impulses. $\rho(t)$ is a continuous function of time with derivative equal to one almost everywhere except at the update times $t_{k}$. We denote its upper bound by $\rho_{\max }:=\sup _{t \geq 0} \rho(t)$, which is a function of $h_{\min }, h_{\max }, \tau_{\min }, \tau_{\max }$. We define $t_{d}:=h_{\max }+\tau_{\max }$, $\left|\boldsymbol{x}_{m}(t)\right|:=\max _{-t_{d} \leq \theta \leq 0}|\boldsymbol{x}(t+\theta)|$, for $t \geq 0$ and $\left\|\boldsymbol{x}_{m}\right\|_{t_{0}}:=$ $\sup _{s \geq t_{0}}\left|\boldsymbol{x}_{m}(s)\right|=\sup _{s \geq t_{0}-t_{d}}|\boldsymbol{x}(s)|$.

\section{Theorem 2}

Assume that there exist $\alpha_{1}, \alpha_{2} \in \mathcal{K}_{\infty}, \gamma_{v}, \gamma_{w} \in \mathcal{G}$, a scalar $\alpha_{3}>0$, and a function $V$ as in (20), such that for any impulse-delay sequence $\left\{s_{k}, \tau_{k}\right\} \in \mathcal{S}$ the corresponding solution $\boldsymbol{x}$ to (19) satisfies:

$$
\begin{aligned}
& \alpha_{1}(|\boldsymbol{x}(t)|) \leq V(t) \leq \alpha_{2}(|\boldsymbol{x}(t)|), \forall \rho \in\left[0, \rho_{\max }\right], \forall t \geq 0 \\
& V(t) \geq \max \left\{\gamma_{v}\left(V_{m}(t)\right), \gamma_{w}\left(\|\boldsymbol{w}\|_{t_{0}}\right)\right\} \\
& \quad \Rightarrow \frac{\mathrm{d} V(t)}{\mathrm{d} t} \leq-\alpha_{3} V(t), \forall t \geq 0 \\
& \gamma_{v}(s)<s, \forall s>0,
\end{aligned}
$$

and that

$$
V\left(t_{k+1}\right) \leq \lim _{t \uparrow t_{k+1}} V(t), \quad \forall k \in \mathbb{N} .
$$

Then, the system (19) is uniformly ISS over the class $\mathcal{S}$ of impulse-delay sequences with $\gamma(s):=\alpha_{1}^{-1}\left(\gamma_{w}(s)\right)$, $\beta(s, t):=\alpha_{1}^{-1}\left(e^{-\left\lfloor\frac{t+t_{d}}{T+t_{d}}\right\rfloor \alpha_{3} T} \alpha_{2}(s)\right)$, where $T>0$ is small enough such that $\gamma_{v}(s) \leq s e^{-\alpha_{3} T} \forall s \leq V_{m}\left(t_{0}\right)$.

Note that $\beta(s, t)$ satisfies all the conditions of a class- $\mathcal{K} \mathcal{L}$ function except that for fixed $s$ it is only non-increasing and not continuous everywhere because for $n\left(T+t_{d}\right) \leq t<$ $(n+1)\left(T+t_{d}\right), \forall n \in \mathbb{N}$ the function $\beta(s, t)$ is flat and it reduces at $t=n(t+T), \forall n \in \mathbb{N}$. However, it is easy to construct a $\bar{\beta}(s, t) \in \mathcal{K} \mathcal{L}$ from $\beta(s, t)$.

System (6) can be written as a delay impulsive system of the form

$$
\begin{aligned}
& \dot{\boldsymbol{\zeta}}(t)=\boldsymbol{F} \boldsymbol{\zeta}(t)+\overline{\boldsymbol{B}}_{2} \boldsymbol{\Delta} \boldsymbol{u}^{f f}(t), t \in\left[t_{k}, t_{k+1}\right) \\
& \boldsymbol{\zeta}\left(t_{k+1}\right)=\left[\begin{array}{ll}
\boldsymbol{e}^{T}\left(t_{k+1}\right) & \boldsymbol{e}^{T}\left(s_{k+1}\right)
\end{array}\right]^{T}, k \in \mathbb{N},
\end{aligned}
$$

with the initial condition $\boldsymbol{\zeta}(0):=\left[\begin{array}{lll}\boldsymbol{e}^{T}(0) & \boldsymbol{e}^{T}\left(s_{0}\right)\end{array}\right]^{T}$, $\boldsymbol{\zeta}(t):=\left[\begin{array}{ll}\boldsymbol{e}^{T}(t) & \boldsymbol{v}_{1}^{T}(t)\end{array}\right]^{T}, \boldsymbol{v}_{1}(t):=\boldsymbol{e}\left(s_{k}\right)$, for $t \in$ $\left[t_{k}, t_{k+1}\right)$, and $\boldsymbol{F}:=\left[\begin{array}{cc}\boldsymbol{A} & \boldsymbol{B}_{1} \\ \mathbf{0} & \mathbf{0}\end{array}\right], \overline{\boldsymbol{B}}_{2}:=\left[\begin{array}{c}\boldsymbol{B}_{2} \\ \mathbf{0}\end{array}\right]$. We employ a Lyapunov candidate function of the form $V(t):=\boldsymbol{e}^{T} \boldsymbol{P} \boldsymbol{e}$ $\left(\rho_{\max }-\rho\right)\left(\boldsymbol{e}-\boldsymbol{v}_{2}\right)^{T} \boldsymbol{X}\left(\boldsymbol{e}-\boldsymbol{v}_{2}\right)$, where $V(t)=V(\tilde{\boldsymbol{\zeta}}(t), \rho(t))$, $\boldsymbol{v}_{2}:=\boldsymbol{e}\left(t_{k}\right), t \in\left[t_{k}, t_{k+1}\right), \tilde{\boldsymbol{\zeta}}:=\left[\begin{array}{ll}\boldsymbol{e}^{T} & \boldsymbol{v}_{2}^{T}\end{array}\right]^{T}$ and $\boldsymbol{P}, \boldsymbol{X}$ are symmetric positive definite matrices. Note that $V(t)$ is positive (for any $\boldsymbol{e}$ and $\boldsymbol{v}_{2}$ not both equal to zero) and satisfies (21). Along jumps this Lyapunov function does not increase since the first term remains unchanged and the second term is non-negative before the jumps and it becomes zero right after the jumps and consequently (24) holds. We choose $\gamma_{v}(s):=p s, 0<p<1$; so (23) holds and we choose $\gamma_{w}(s):=g_{w} s^{2}, g_{w}>0$. If the LMIs that appear below in Theorem 3 are feasible then (22) is satisfied and consequently Theorem 2 guarantees that system (25) is uniformly ISS over the class $\mathcal{S}$ of sampling-delay sequences.

\section{Theorem 3}

Assume that there exist positive scalars $\alpha, \lambda_{i}, 1 \leq i \leq$ $4, g_{w}, p<1$ and symmetric positive definite matrices $\boldsymbol{P}, \boldsymbol{X}$ and (not necessarily symmetric) matrices $N_{1}, N_{2}$ that satisfy the following LMIs:

$$
\left[\begin{array}{cccc}
\boldsymbol{M}_{1}+\rho_{\max } \boldsymbol{M}_{2} & \boldsymbol{N}_{1} \boldsymbol{A} & \boldsymbol{N}_{1} \boldsymbol{B}_{1} & \boldsymbol{N}_{1} \boldsymbol{B}_{2} \\
* & -\tau_{\max }^{-1} \lambda_{1} \boldsymbol{P} & \mathbf{0} & \mathbf{0} \\
* & * & -\tau_{\max }^{-1} \lambda_{3} \boldsymbol{P} & 0 \\
* & * & * & -\tau_{\max }^{-1} \lambda_{2} \boldsymbol{I}
\end{array}\right]<0,
$$


$\left[\begin{array}{ccc}\boldsymbol{M}_{1}+\rho_{\max } \boldsymbol{M}_{3} & \boldsymbol{N}_{1} \boldsymbol{A} & \boldsymbol{N}_{1} \boldsymbol{B}_{1} \\ * & -\tau_{\max }^{-1} \lambda_{1} \boldsymbol{P} & \mathbf{0} \\ * & * & -\tau_{\max }^{-1} \lambda_{3} \boldsymbol{P} \\ * & * & * \\ * & * & * \\ * & * & *\end{array}\right.$

$$
\left.\begin{array}{ccc}
\boldsymbol{N}_{1} \boldsymbol{B}_{2} & \left(\boldsymbol{N}_{1}+\boldsymbol{N}_{2}\right) A & \left(\boldsymbol{N}_{1}+\boldsymbol{N}_{2}\right) \boldsymbol{B}_{2} \\
\mathbf{0} & \mathbf{0} & \mathbf{0} \\
\mathbf{0} & \mathbf{0} & \mathbf{0} \\
-\tau_{\max }^{-1} \lambda_{2} \boldsymbol{I} & \mathbf{0} & \mathbf{0} \\
* & -\rho_{\max }^{-1} \lambda_{1} \boldsymbol{P} & \mathbf{0} \\
* & * & -\rho_{\max }^{-1} \lambda_{2} \boldsymbol{I}
\end{array}\right]
$$

where

$$
\begin{aligned}
& \beta_{1}:=\left(\lambda_{1}+\lambda_{2} g_{w}^{-1}\right) \tau_{\max } p+\lambda_{4}+\alpha, \\
& \overline{\boldsymbol{F}}:=\left[\begin{array}{llll}
\boldsymbol{A} & \boldsymbol{B}_{1} & \mathbf{0} & \boldsymbol{B}_{2}
\end{array}\right], \beta_{2}:=\lambda_{1} p+\lambda_{2} g_{w}^{-1} p, \\
& \boldsymbol{M}_{1}:=\left[\begin{array}{l}
\boldsymbol{P} \\
\mathbf{0} \\
\mathbf{0} \\
\mathbf{0}
\end{array}\right] \overline{\boldsymbol{F}}+\overline{\boldsymbol{F}}^{T}\left[\begin{array}{l}
\boldsymbol{P} \\
\mathbf{0} \\
\mathbf{0} \\
\mathbf{0}
\end{array}\right]^{T}-\boldsymbol{N}_{1}\left[\begin{array}{llll}
\boldsymbol{I} & -\boldsymbol{I} & \mathbf{0} & \mathbf{0}
\end{array}\right] \\
& -\left[\begin{array}{llll}
\boldsymbol{I} & -\boldsymbol{I} & \mathbf{0} & \mathbf{0}
\end{array}\right]^{T} \boldsymbol{N}_{1}^{T}-\boldsymbol{N}_{2}\left[\begin{array}{lllll}
\boldsymbol{I} & \mathbf{0} & -\boldsymbol{I} & \mathbf{0}
\end{array}\right] \\
& -\left[\begin{array}{llll}
\boldsymbol{I} & 0 & -\boldsymbol{I} & \mathbf{0}
\end{array}\right]^{T} \boldsymbol{N}_{2}^{T}-\left[\begin{array}{c}
\boldsymbol{I} \\
\mathbf{0} \\
-\boldsymbol{I} \\
\mathbf{0}
\end{array}\right] \boldsymbol{X}\left[\begin{array}{llll}
\boldsymbol{I} & \mathbf{0} & -\boldsymbol{I} & \mathbf{0}
\end{array}\right] \\
& -\lambda_{4} g_{w}\left[\begin{array}{l}
\mathbf{0} \\
\mathbf{0} \\
\mathbf{0} \\
\boldsymbol{I}
\end{array}\right]\left[\begin{array}{llll}
\mathbf{0} & \mathbf{0} & \mathbf{0} & \boldsymbol{I}
\end{array}\right]+\beta_{1}\left[\begin{array}{l}
\boldsymbol{P} \\
\mathbf{0} \\
\mathbf{0} \\
\mathbf{0}
\end{array}\right]\left[\begin{array}{llll}
\boldsymbol{I} & \mathbf{0} & 0 & \mathbf{0}
\end{array}\right] \\
& +\lambda_{3} p \tau_{\max }\left[\begin{array}{l}
\mathbf{0} \\
\mathbf{0} \\
\boldsymbol{P} \\
\mathbf{0}
\end{array}\right]\left[\begin{array}{llll}
\mathbf{0} & \mathbf{0} & \boldsymbol{I} & \mathbf{0}
\end{array}\right] \text {, } \\
& \boldsymbol{M}_{2}:=\left[\begin{array}{c}
\boldsymbol{I} \\
\mathbf{0} \\
-\boldsymbol{I} \\
\mathbf{0}
\end{array}\right] \boldsymbol{X} \overline{\boldsymbol{F}}+\overline{\boldsymbol{F}}^{T} \boldsymbol{X}\left[\begin{array}{c}
\boldsymbol{I} \\
\mathbf{0} \\
-\boldsymbol{I} \\
\mathbf{0}
\end{array}\right]^{T} \\
& +\left(\beta_{1}+\beta_{2} \rho_{\max }\right)\left[\begin{array}{c}
\boldsymbol{I} \\
\mathbf{0} \\
-\boldsymbol{I} \\
\mathbf{0}
\end{array}\right] \boldsymbol{X}\left[\begin{array}{c}
\boldsymbol{I} \\
\mathbf{0} \\
-\boldsymbol{I} \\
\mathbf{0}
\end{array}\right]^{T}, \\
& \boldsymbol{M}_{3}:=\beta_{2}\left[\begin{array}{l}
\boldsymbol{P} \\
\mathbf{0} \\
\mathbf{0} \\
\mathbf{0}
\end{array}\right]\left[\begin{array}{llll}
\boldsymbol{I} & 0 & 0 & 0
\end{array}\right]+\left(\boldsymbol{N}_{1}+\boldsymbol{N}_{2}\right) \boldsymbol{B}_{1}\left[\begin{array}{llll}
\mathbf{0} & \boldsymbol{I} & \mathbf{0} & \mathbf{0}
\end{array}\right] \\
& +\left[\begin{array}{l}
\mathbf{0} \\
\boldsymbol{I} \\
\mathbf{0} \\
\mathbf{0}
\end{array}\right] \boldsymbol{B}_{1}^{T}\left(\boldsymbol{N}_{1}+\boldsymbol{N}_{2}\right)^{T} .
\end{aligned}
$$

Then, system (6) is uniformly ISS over the class $\mathcal{S}$ of sampling-delay sequences with respect to the time-varying input $\boldsymbol{\Delta} \boldsymbol{u}^{f f}(t)$, i.e., inequality (7) is satisfied with the functions $\beta, \gamma$ defined in (14) with

$$
g_{2}:=\sqrt{\frac{g_{w}}{\lambda_{\min }(P)}}, g_{1}(t):=\sqrt{\left.\frac{\lambda_{\max }(P)}{\lambda_{\min }(P)} p^{\left\lfloor\frac{\alpha\left(t+t_{d}\right)}{\alpha t}\right\rfloor}\right\rfloor} .
$$

The conditions in Theorem 3 depend on $\tau_{\max }$ and $\rho_{\max }$ which is the maximum of the input update interval. With regard to the fact that $\rho_{\max } \leq \tau_{\max }+h_{\max }$, we can replace $\rho_{\max }$ by $\tau_{\max }+h_{\max }$ and express the conditions in Theorem 3 in terms of $\tau_{\max }$ and $h_{\max }$. However, these conditions do not explicitly depend on the values of $h_{\min }$ and $\tau_{\text {min }}$. Consequently, this approach towards modeling NCSs may result in more conservative conditions in comparison to those obtained using the discrete-time approach when $0 \ll h_{\min } \simeq h_{\max }$ or $0 \ll \tau_{\min } \simeq \tau_{\max }$. These topics will be discussed further in the examples presented in Section V.

\section{Tracking Control Performance}

Theorems 1 and 3 on the ISS property of NCSs can be applied to the tracking problem of NCSs with variable sampling intervals and delays, as stated in Section II. Namely, the satisfaction of the conditions in either of these theorems guarantees that the approximate tracking problem is solved and an ultimate bound on the tracking error is available. A straightforward analysis shows that an upper bound on the feedforward error is given by

$$
\left|\boldsymbol{\Delta} \boldsymbol{u}^{f f}(t)\right| \leq R:=\sqrt{\sum_{i=1}^{m} R_{i}^{2}}, \forall t \in \mathbb{R},
$$

with $R_{i}:=\gamma_{1, i}\left(\tau_{\max }+h_{\max }\right), \quad \gamma_{1, i}=\sup _{t \in \mathbb{R}}\left|\frac{\partial u_{e, i}^{f f}(t)}{\partial t}\right|$ where $u_{e, i}^{f f}$ denotes the $i$-th component of $\boldsymbol{u}_{e}^{f f}$, for $i=$ $1, \ldots, m$. The following corollary is based on Theorems 1 and 3 and the bound on the feedforward error defined in (30). It characterizes the steady-state tracking performance achieved by applying the tracking controller (3), with $\boldsymbol{u}_{e}^{f f}(t)$ satisfying (2), to the NCS (1).

\section{Corollary 1}

Consider the NCS (1), with sampling-delay sequences $\left\{s_{k}, \tau_{k}\right\} \in \mathcal{S}$ and $\mathcal{S}$ defined by (8). Consider controller (3), with $\boldsymbol{u}_{e}^{f f}(t)$ satisfying (2). If either the LMIs (13) or the LMIs (26)-(28) are feasible, then the tracking error dynamics (6) is uniformly ISS with respect to the feedforward error $\Delta \boldsymbol{u}^{f f}(t)$ over the class $\mathcal{S}$. Moreover, the tracking error is globally uniformly ultimately bounded with the asymptotic bound computed from the following methods:

- Method 1: if the LMIs (13) are feasible, then the asymptotic bound on the tracking error is given by $\limsup _{t>0}|\boldsymbol{e}(t)| \leq g_{2} R$, with $g_{2}$ given in (15) and $R$ given in (30);

- Method 2: if the LMIs (26)-(28) are feasible, then the asymptotic bound on the tracking error is given by $\limsup _{t>0}|\boldsymbol{e}(t)| \leq g_{2} R$, with $g_{2}$ given in (29) and $R$ given in (30).

\section{Illustrative EXAMPLE}

We consider an example of a motion control system from the document printing domain. The continuous-time statespace representation can be described by (1), with $\boldsymbol{A}=$ $\left(\begin{array}{ll}0 & 1 \\ 0 & 0\end{array}\right), \boldsymbol{B}=\left(\begin{array}{l}0 \\ b\end{array}\right)$, with $b=\frac{n r_{R}}{J_{M}+n^{2} J_{R}}$. Herein, the first state represents the sheet position (of a sheet in a single motor-roller pair) and the second state is the sheet velocity. Moreover, $J_{M}=1.95 \cdot 10^{-5} \mathrm{kgm}^{2}$ the inertia of the motor, $J_{R}=6.5 \cdot 10^{-5} \mathrm{kgm}^{2}$ the inertia of the roller, $r_{R}=14 \cdot 10^{-3}$ $\mathrm{m}$ the radius of the roller, $n=0.2$ the transmission ratio between motor and roller and $u$ the motor torque. The feedback gain matrix in (3) is $K=\left(\begin{array}{ll}50 & 1.18\end{array}\right)$. Consider a harmonic desired trajectory: $\boldsymbol{x}_{d}(t)=\left(\begin{array}{lll}A_{d} \sin (\omega t) & A_{d} \omega \cos (\omega t)\end{array}\right)^{T}$, with $A_{d}=0.01$ and $\omega=2 \pi$. The exact feedforward is given by $u_{e}^{f f}(t)=-\frac{A_{d} \omega^{2}}{b} \sin (\omega t)$.

Let us first consider the case of a constant sampling interval ( $h=5 \times 10^{-3} \mathrm{~s}$ ), but time-varying and uncertain 


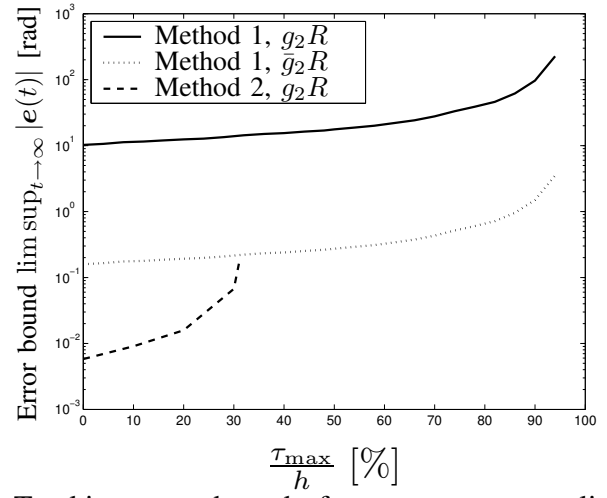

Figure 3: Tracking error bounds for a constant sampling interval $h=5 \times 10^{-3} \mathrm{~s}$ and time-varying and uncertain delays in the set $\left[0, \tau_{\max }\right]$.

delays in the set $\left[0, \tau_{\max }\right]$. Figure 3 depicts the error bounds as provided in Corollary 1 for $\tau_{\max }<h$. The results for the discrete-time approach are obtained using a finite set of LMIs guaranteeing the satisfaction of (13), based on a (real) Jordan form approach as in [5]. Note that, for the discretetime modeling approach, also the bound for the tracking error at the sampling times $s_{k}\left(\bar{g}_{2} R\right.$, with $\bar{g}_{2}$ as in (18)) is included by means of the dotted line. Figure 3 shows that by using the discrete time approach, ISS can be guaranteed up to $\tau_{\max }=0.94 h$, but using the delay impulsive approach ISS can be guaranteed only up to $\tau_{\max }=0.33 \mathrm{~h}$. So, the discrete-time approach allows to prove ISS for a larger range of delays. However, the delay impulsive modeling/analysis approach provides much tighter (ISS) bounds on the tracking error (note that the scale of the vertical axis is logarithmic). Note that the overestimation of the bound on the tracking error for the discrete-time modeling approach is significantly worsened due to upperbounding the intersample behavior (compare the solid and dotted lines in Figure 3).

Next, we consider the case in which the sampling interval is variable, i.e., $h \in\left[h_{\min }, h_{\max }\right]$, and the delay is zero. Figure 4 depicts the error bounds as provided in Corollary 1. In this example, we take $h_{\text {min }}=h_{\text {max }} / 1.5$, so $h_{\text {min }} \neq 0$. Using the discrete-time modeling approach, we can assure ISS almost up to $h_{\max }=1.34 \times 10^{-2} \mathrm{~s}$, which is the sampling interval for which the system with a constant sampling interval (and no delay) becomes unstable (see the dashed vertical line in Figure 4). This fact shows that the proposed ISS conditions are not conservative from a stability perspective. Using the delay impulsive modeling approach, ISS can only be guaranteed up to $h_{\max }=9 \times 10^{-3} \mathrm{~s}$. However, the delay impulsive approach clearly provides significantly less conservative bounds on the tracking error. This type of plot is instrumental in determining an upperbound on the maximum sampling interval needed to guarantee a minimum level of steady-state tracking performance.

\section{CONCLUSIONS}

In this paper, a solution to the approximate tracking problem of Networked Control Systems (NCSs) with uncertain, time-varying sampling intervals and network delays is presented. The uncertain, time-varying sampling intervals and delays cause inexact feedforward, which induces a perturbation on the tracking error dynamics.

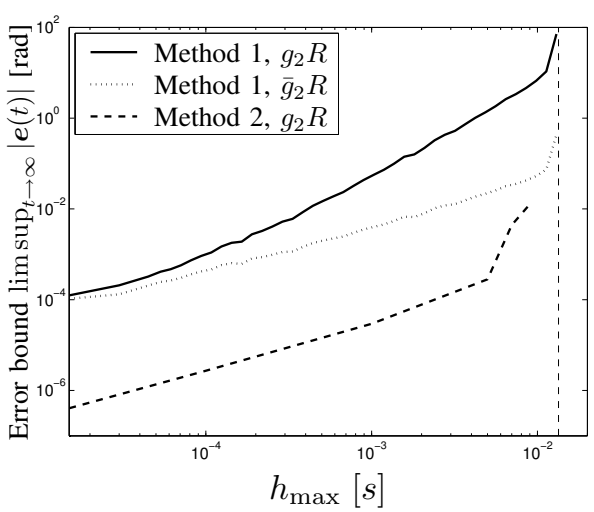

Figure 4: Tracking error bounds for variable sampling intervals $h \in$ $\left[h_{\min }, h_{\max }\right]$ and no delays, where $h_{\max }=1.5 h_{\min }$.

Sufficient conditions in terms of LMIs for the input-tostate stability (ISS) of the tracking error dynamics with respect to this perturbation are given. Hereto, two NCS modeling approaches are used: a discrete-time model and a model in terms of delay impulsive differential equations. These ISS results provide bounds on the steady-state tracking error as a function of the plant properties, the controller parameters and the network properties. Such error bounds can readily be used to formulate design rules regarding the maximum sampling interval or the maximum delay allowed to guarantee a certain steady-state tracking performance.

The results are illustrated on a mechanical motion control problem showing the effectiveness of the proposed strategy and providing insight in the differences and commonalities between the two NCS modeling approaches.

\section{REFERENCES}

[1] B. W. Zhang, M. S. Branicky, and S. M. Phillips, "Stability of networked control systems," IEEE Control Systems Magazine, vol. 21, no. 1, pp. 84-99, February 2001.

[2] T. C. Yang, "Networked control system: a brief survey," IEE Proc.Control Theory Appl., vol. 153, no. 4, pp. 403-412, July 2006.

[3] B. Wittenmark, J. Nilsson, and M. Törngren, "Timing problems in realtime control systems," in Proc. of the American Control Conference, Seattle, Washington, USA, 1995, pp. 2000-2004.

[4] J. Nilsson, Real-Time Control Systems with Delays. Lund, Sweden: $\mathrm{PhD}$ thesis, Dept. of Automatic Control, Lund Inst. of Techn., 1998.

[5] M. Cloosterman, N. van de Wouw, W. Heemels, and H. Nijmeijer, "Stability of networked control systems with large delays," in Proc. of the 46th IEEE Conf. on Decision and Control, New Orleans, 2007.

[6] L. Hetel, J. Daafouz, and C. Iung, "Stabilization of switched linear systems with unknown time varying delays," in Proc. of the 2nd IFAC conf. on Anal. and Design of Hybrid Syst., Italy, 2006, pp. 335-340.

[7] P. Naghshtabrizi and J. Hespanha, "Stability of network control systems with variable sampling and delays," in Proc. of the 44th Annual Allerton Conf. on Comm., Control, and Computing, 2006.

[8] H. Gao and T. Chen, " $H_{\infty}$ model reference control for networked feedback systems," in Proceedings of the 45th IEEE Conference on Decision and Control, San Diego, 2006.

[9] S. Yüksel, H. Hindi, and L. Crawford, "Optimal tracking with feedback-feedforward control separation over a network," in Proceedings of American Control Conference 2006, Minneapolis, U.S.A., 2006.

[10] D. Nesic and A. Teel, "Input-to-state stability of networked control systems," Automatica, vol. 40, no. 12, pp. 2121-2128, 2004.

[11] N. van de Wouw, P. Naghshtabrizi, M. Cloosterman, and J. Hespanha, "Tracking control for networked control systems," Eindhoven University of Technology, The Netherlands, Tech. Rep. DCT2007.116, 2007.

[12] Y. Yang, D. Xu, M. Tan, and X. Dai, "Stochastic stability analysis and control of networked control systems with randomly varying long time-delays," in Proc. of the 5th World Congress on Intelligent Control and Automation, Hangzhou, China, June 2004, pp. 1391-1395.

[13] A. Teel, "Connections between Razumikhin-type theorems and the ISS nonlinear small gain theorem," IEEE Transactions on Automatic Control, vol. 43, pp. 960-964, 1998. 